\title{
Three-photon bimolecular absorption
}

\author{
David L Andrews and Alexander M Bittner \\ School of Chemical Sciences, University of East Anglia, Norwich NR4 7TJ, UK
}

Received 15 June 1992, in final form 11 November 1992

\begin{abstract}
Three-photon bimolecular absorption is a process which, through the simultaneous absorption of three laser photons, results in the electronic excitation of two molecules or atoms. Following a recent treatment of the selection rules, a quantum electrodynamical treatment is employed to describe the main features associated with the process and to produce an equation for the excitation rate. The dependence on photon polarization is investigated, and the magnitude of the excitation rate is estimated. It is shown that two different mechanisms operate, each with different selection rules and different dependences on intermolecular separation. In each case an energy mismatch is conveyed from one molecule to the other by means of virtual photon coupling. Some possible schemes for experimental study of the process are outlined.
\end{abstract}

\section{Introduction}

Bimolecular photoabsorption is a process which leads to the simultaneous electronic excitation of two molecules (or atoms). It is also known as synergistic, cooperative, collective or pair absorption. The excitation energy is generally provided by the absorption of one or two simultaneously absorbed photons. Both experiment and theory are well developed, and the two-photon case has been reviewed recently (Andrews and Hopkins 1990). It has also recently been suggested that one-photon processes of this type might account for features in the water vapour absorption continuum (Hudis et al 1991, 1992).

Although three-photon absorption in individual molecules and atoms is well documented, bimolecular three-photon absorption has not yet been observed. However, a recent treatment of the selection rules for bimolecular absorption has included a study of the three-photon case and identified possible systems for its observation (Andrews and Bittner 1992). In this paper a quantum electrodynamical treatment is employed to describe the features associated with the process and to produce an equation for the excitation rate.

In the process to be considered three photons of circular frequency $\omega$ from a single laser beam have to provide a sum of the excitation energies for two molecules $A$ and $\mathrm{B}$ (here and subsequently the term molecule may also signify an atom):

$$
\mathrm{A}+\mathrm{B}+3 \hbar \omega \rightarrow \mathrm{A}^{*}+\mathrm{B}^{*} \text {. }
$$

The corresponding energy levels are depicted in figure 1. To suppress conventional one-centre transitions, if they are not symmetry-forbidden, neither molecule should possess energy levels at multiples of the photon energy, $\hbar \omega, 2 \hbar \omega$ or $3 \hbar \omega$. 


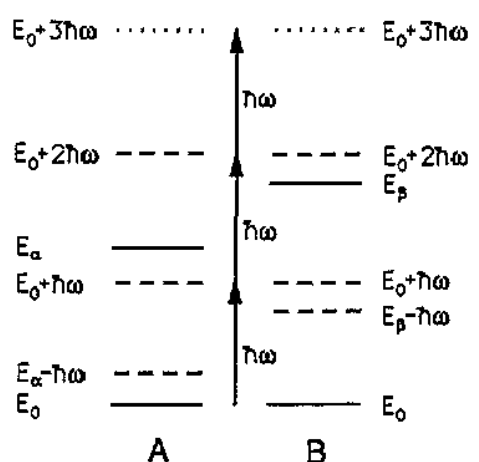

Figure 1. Energy levels involved in three-photon bimolecular absorption. The broken lines correspond to resonances which apply to both mechanisms (see section 6). The dotted line applies only to the $(4,1)$ mechanism.

The basis for quantum electrodynamical calculations of non-linear optical effects comprises setting up time-ordered diagrams (Wallace 1966). The 160 diagrams describing three-photon bimolecular absorption imply that two different mechanisms with different selection rules exist. It is shown that these mechanisms also differ when their dependence on the spatial separation $R$ of $A$ and $B$ is considered. The first one entails the absorption of all three photons at one molecule; the second is associated with two-photon absorption at one and one-photon absorption at the other molecule. None of the absorption processes is energy conserving at either individual centre A or B; thus in each case an energy mismatch has to be conveyed between the pair. This is achieved by virtual photon coupling.

For freely rotating molecules in a fluid sample a triple rotational average of the rate equation has to be performed (Schipper 1981). The condition of two molecular centres in a fixed mutual orientation in an otherwise fluid sample requires a single rotational average. Rate equations for each of these situations are explicitly computed for the most important case where the intermolecular distance is much smaller than the photon wavelength. The changes in absorption associated with modifying the laser beam polarization are also investigated. Finally the magnitude of the excitation rate is estimated and compared to the rate of one-centre three-photon absorption.

\section{Derivation of rate equations}

The aim of the calculation is to find an equation for the absorption rate, expressed in a form which is readily interpretable. The starting point is the Fermi golden rule,

$$
\Gamma=2 \pi \rho_{f}\left|M^{f i}\right|^{2} / \hbar
$$

where $\Gamma$ stands for the absorption rate and $M^{f i}$ is the quantum electrodynamical matrix element for the process. Here $\rho_{f}$ is the density of the final states, given by a convolution of the final state densities for $A$ and $B$ :

$$
\rho_{f}=\int_{0}^{\infty} \rho_{f}^{\mathrm{A}}(E) \rho_{f}^{\mathrm{B}}(3 \hbar \omega-E) \mathrm{d} E .
$$

The matrix element is calculated from time-dependent perturbation theory with the aid of time-ordered diagrams, each of which represents a contribution to the quantum 
probability amplitude for the overall process. The detailed basis for such calculations is described more fully elsewhere (Craig and Thirunamachandran 1984, Andrews et al 1989). The diagrams to be employed here are constructed on the basis of neglecting wavefunction overlap between the molecules $\mathrm{A}$ and $\mathrm{B}$, as is reflected in their separate state lines in the diagrams. It is important to note that in the Power-Zienau-Woolley formulation of quantum electrodynamics there are no interactions between molecules other than those mediated by the electromagnetic field, i.e. by the exchange of virtual photons (Power and Zienau 1959, Woolley 1971).

In the most general case of three-photon bimolecular absorption 480 time-ordered diagrams should be constructed. We can divide them into two types defining two mechanisms, first where all three photons are absorbed at one molecule, secondly where two photons are absorbed at one and a third at the other molecule. There are 240 diagrams of the first type, reducing in the case of single-beam excitation to $240 / 3 !=40$ because the three photons are indistinguishable. Similarly the 240 diagrams of the second type reduce to $240 / 2 !=120$ in the single-beam case. Of these, half in each case are mirror images of the other half, in which the roles of $A$ and $B$ are interchanged. Examples are shown in figure 2. In each diagram the fourth photon propagating between $\mathrm{A}$ and $\mathrm{B}$ is a virtual photon which conveys the energy mismatch. So in three-photon bimolecular photoabsorption altogether five molecule-photon interactions occur, two of them involving the virtual photon. The possible partitions of this number, $(4,1)$ and $(3,2)$, designate the two mechanisms.

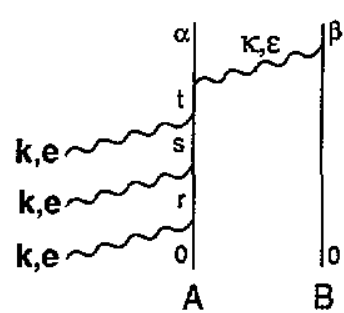

(a)

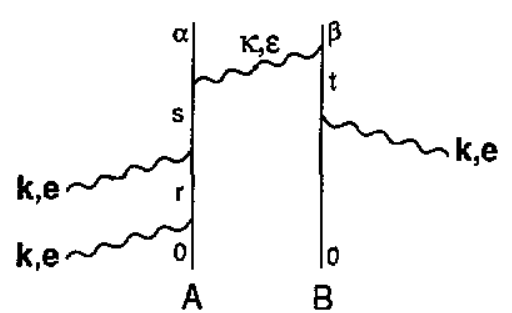

(b)

Figure 2. Typical time-ordered diagrams for three-photon bimolecular absorption; (a) $(4,1)$ mechanism; (b) $(3,2)$ mechanism.

The absorptions taking place at either individual molecule are not energy conserving as it is assumed that there is no excited state at energy $\hbar \omega, 2 \hbar \omega$ or $3 \hbar \omega$. Virtual photons are not energy conserving either. However, it is important to take account of the overall energy conservation:

$$
E_{\alpha 0}+E_{\beta 0}=3 \hbar \omega .
$$

Here $E_{\alpha 0}$ and $E_{\beta 0}$ denote the energy differences between the ground and excited state within $A$ and $B$, respectively.

Each molecule-radiation interaction in the time-ordered diagrams is assumed to occur via electric-dipole coupling, yielding the interaction Hamiltonian

$$
H_{\mathrm{int}}=-\varepsilon_{0}^{-1} \mu \cdot d^{\perp}
$$

where $\boldsymbol{\mu}$ denotes the appropriate molecular dipole moment operator and $\boldsymbol{d}^{\perp}$ the transverse electric displacement operator of the radiation field. 
Since five interactions occur altogether, fifth-order perturbation theory is used to compute each matrix element. Using the quantum electrodynamical form of $d^{L}$ expressed in terms of annihilation and creation operators, the matrix elements pertaining to the time-ordered diagrams in figure 2 are given by

$$
\begin{aligned}
& M_{1}^{(4,1)}=\mathrm{i}(\hbar c)^{5 / 2}\left(2 \varepsilon_{0} V\right)^{-5 / 2} \sum_{\kappa} \sum_{\varepsilon} k^{3 / 2} \kappa n^{3 / 2} e_{i} e_{j} e_{k} \bar{\varepsilon}_{i} \varepsilon_{m} \\
& \times \exp \left(3 \mathrm{i} k \cdot \boldsymbol{R}_{\mathrm{A}}+\mathrm{i} \boldsymbol{\kappa} \cdot \boldsymbol{R}\right) \sum_{r} \sum_{s} \sum_{t}^{\mathrm{A}} \mu_{t}^{r 0 \mathrm{~A}} \mu_{j}^{s r \mathrm{~A}} \mu_{k}^{z s \mathrm{~A}} \mu_{l}^{\alpha z \mathrm{~B}} \mu_{m}^{\beta 0} \\
& \times\left(E_{0 r}+\hbar \omega\right)^{-1}\left(E_{0 s}+2 \hbar \omega\right)^{-1}\left(E_{\alpha t}+E_{\beta 0}\right)^{-1}\left(E_{\beta 0}-\hbar c \kappa\right)^{-1} \\
& M_{1}^{(3,2)}=\mathrm{i}(\hbar c)^{5 / 2}\left(2 \varepsilon_{0} V\right)^{-5 / 2} \sum_{k} \sum_{\varepsilon} k^{3 / 2} \kappa n^{3 / 2} e_{i} e_{j} e_{k} \bar{\varepsilon}_{l} \varepsilon_{m} \\
& \times \exp \left[3 i k \cdot\left(\boldsymbol{R}_{\mathrm{A}}+\boldsymbol{R}\right)+\mathrm{i} \boldsymbol{\kappa} \cdot \boldsymbol{R}\right] \sum_{r} \sum_{s} \sum_{t}^{\mathrm{A}} \mu_{i}^{r 0 \mathrm{~A}} \mu_{j}^{s \boldsymbol{B}} \mu_{k}^{\text {to } \mathrm{A}} \mu_{l}^{\alpha s \mathrm{~B}} \mu_{m}^{\beta t} \\
& \times\left(E_{0 r}+\hbar \omega\right)^{-1}\left(E_{0 s}+2 \hbar \omega\right)^{-1}\left(E_{\alpha s}+E_{\beta t}\right)^{-1}\left(E_{\beta t}-\hbar c \kappa\right)^{-1} .
\end{aligned}
$$

The symbols used are: $V$ for the quantization volume, $k$ for the wavevector of the laser beam, $\boldsymbol{\kappa}$ for the wavevector of the virtual photon, $n$ for the number of real photons present in the quantization volume ( $n \gg 1$ is implied), $e$ and $\varepsilon$ for the polarization vectors of real and virtual photons, $\boldsymbol{R}_{\mathrm{A}}$ for the position of molecule $\mathrm{A}$, $\boldsymbol{R}=\boldsymbol{R}_{\mathrm{B}}-\boldsymbol{R}_{\mathrm{A}}$ for the distance vector between $\mathrm{A}$ and $\mathrm{B}$. The implied summation convention is used for the indices. The summed intermediate states $r$ of molecule $A$ are associated with energies $E_{r 0}$ and transition dipole moments ${ }^{\mathrm{A}} \mu^{r 0}$; similar remarks apply to the other intermediate states $s$ and $t$ and to molecule $\mathrm{B}$.

The matrix element $M^{(4,1)}$ comprises a sum of $M_{1}^{(4,1)}$ and all other 39 matrix elements for the $(4,1)$ case. Similarly $M^{(3,2)}$ contains 120 terms. These contributions are summed by a method which is outlined in the appendix, where the full results for $M^{(4,1)}$ and $M^{(3,2)}$ are also presented. Inserting these in the rate equation (2) then gives the following results for $\Gamma^{(4,1)}$ and $\Gamma^{(3,2)}$;

$$
\begin{aligned}
& \Gamma^{(4,1)}=\pi \rho_{f} I^{3}\left(4 \hbar c^{3} \varepsilon_{0}^{3}\right)^{-1} \\
& \times\left\{e _ { i } e _ { j } e _ { k } \overline { e } _ { n } \overline { e } _ { o } \overline { e } _ { p } \left[T_{(i j k) l}^{\alpha 0} \bar{T}_{(\text {nop }) q}^{\alpha 0} \mu_{m}^{\beta 0} \bar{\mu}_{r}^{\beta 0} V_{(l m)}\left(\omega_{\beta 0}, \boldsymbol{R}\right) \bar{V}_{(q r)}\left(\omega_{\beta 0}, \boldsymbol{R}\right)\right.\right. \\
& \left.+T_{(y k) l}^{\beta 0} \bar{T}_{(n o p) q}^{\beta 0} \mu_{m}^{\alpha 0} \bar{\mu}_{r}^{\alpha 0} V_{(I m)}\left(\omega_{\alpha 0}, R\right) \bar{V}_{(q r)}\left(\omega_{\alpha 0}, R\right)\right] \\
& +2 \operatorname{Re}\left[e_{t} e_{j} e_{k} \bar{e}_{n} \bar{e}_{o} \bar{e}_{p} T_{(j k) l}^{\alpha 0} \bar{T}_{(\text {nop }) q}^{\beta 0} \mu_{m}^{\beta 0} \bar{\mu}_{r}^{\alpha 0} V_{(l m)}\left(\omega_{\beta 0}, R\right)\right. \\
& \left.\left.\times \vec{V}_{(q r)}\left(\omega_{\alpha 0}, \boldsymbol{R}\right) \exp (-3 i k \cdot R)\right]\right\} \\
& \Gamma^{(3,2)}=\pi \rho_{f} I^{3}\left(4 \hbar c^{3} \varepsilon_{0}^{3}\right)^{-1}\left\{e_{1} e_{j} e_{k} \bar{e}_{n} \bar{e}_{u} \bar{e}_{p}\right. \\
& \times\left[\chi_{(i j) l}^{\alpha 0} \bar{\chi}_{(n o) q}^{\alpha 0} S_{k m}^{\beta 0} \bar{S}_{p r}^{\beta 0} V_{(l m)}\left(\omega_{\beta 0}-\omega, R\right) \bar{V}_{(q))}\left(\omega_{\beta 0}-\omega, \boldsymbol{R}\right)\right. \\
& \left.+\chi_{(j)) l}^{\beta 0} \bar{\chi}_{(n o) q}^{\beta 0} S_{k m}^{\alpha 0} \bar{S}_{p r}^{\alpha 0} V_{(I m)}\left(\omega_{\alpha 0}-\omega, R\right) \bar{V}_{(q r)}\left(\omega_{\alpha 0}-\omega, R\right)\right] \\
& +2 \operatorname{Re}\left[e_{i} e_{j} e_{k} \bar{e}_{n} \bar{e}_{o} \bar{e}_{p} \chi_{(i j) l}^{\alpha 0} \bar{\chi}_{(n o) q}^{\beta 0} S_{k m}^{\beta 0} \bar{S}_{p r}^{\alpha 0} V_{(I m)}\left(\omega_{\beta 0}-\omega, \boldsymbol{R}\right)\right. \\
& \text { - } \left.\left.\quad \times \overline{\boldsymbol{V}}_{(q r)}\left(\omega_{\alpha 0}-\omega, \boldsymbol{R}\right) \exp (-2 \mathrm{i} \boldsymbol{k} \cdot \boldsymbol{R})\right]\right\}
\end{aligned}
$$

where $I=n \hbar c^{2} k / V$ is the irradiance of the laser beam, $\omega_{\alpha 0}=E_{\alpha 0} / \hbar, \omega_{\beta 0}=E_{\beta 0} / \hbar$ and

$$
\begin{aligned}
V_{(i j)}(\omega, \boldsymbol{R})= & \hbar c\left(2 \varepsilon_{0} V\right)^{-1} \sum_{\kappa} \sum_{\varepsilon} \kappa \varepsilon_{i} \bar{\varepsilon}_{j} \\
& \times\left[\exp (\mathrm{i} \kappa \cdot R)(\hbar \omega-\hbar c \kappa)^{-1}-\exp (-\mathrm{i} \kappa \cdot R)(\hbar \omega+\hbar c \kappa)^{-1}\right] \\
= & \left(4 \pi \varepsilon_{0} R^{3}\right)^{-1} \exp (\mathrm{i} \omega R / c) \\
& \times\left[\left(\delta_{l j}-3 \hat{R}_{i} \hat{R}_{j}\right)(1-\mathrm{i} \omega R / c)-\left(\delta_{i j}-\hat{R}_{i} \hat{R}_{j}\right)(\omega R / c)^{2}\right]
\end{aligned}
$$


is the virtual photon coupling tensor (which is index symmetric, as denoted by the brackets around the indices, and also symmetric with respect to inversion of $\boldsymbol{R})+: \hat{\boldsymbol{R}}$ is the unit displacement vector of B with respect to A. In passing we note that only $e$ and $V_{(i j)}$ can take complex values, all other parameters are real. The molecular tensors are defined by

$$
\begin{aligned}
& S_{i j}^{\alpha 0}=\sum_{r}\left[{ }^{\mathrm{A}} \mu_{i}^{\alpha r \mathrm{~A}} \mu_{J}^{r 0}\left(E_{\alpha r}-\hbar \omega\right)^{-1}+{ }^{\mathrm{A}} \mu_{i}^{r 0 \mathrm{~A}} \mu_{j}^{\alpha r}\left(E_{0 r}+\hbar \omega\right)^{-1}\right] \\
& \chi_{i j h}^{\alpha 0}=\sum_{r} \sum_{s}\left[{ }^{A} \mu_{i}^{r 0 \mathrm{~A}} \mu_{j}^{s r \mathrm{~A}} \mu_{k}^{\alpha s}\left(E_{0 r}+\hbar \omega\right)^{-1}\left(E_{0 s}+2 \hbar \omega\right)^{-1}\right. \\
& +{ }^{\mathrm{A}} \mu_{r}^{r \mathrm{AA}} \mu_{J}^{\alpha s \mathrm{~A}} \mu_{k}^{s r}\left(E_{0 \mathrm{r}}+\hbar \omega\right)^{-1}\left(E_{\alpha s}-\hbar \omega\right)^{-1} \\
& \left.+{ }^{\mathrm{A}} \mu_{i}^{s r \mathrm{~A}} \mu_{j}^{\alpha s \mathrm{~A}} \mu_{k}^{r 0}\left(E_{\alpha r}-2 \hbar \omega\right)^{-1}\left(E_{\alpha s}-\hbar \omega\right)^{-1}\right] \\
& T_{i j k l}^{\alpha 0}=\sum_{r} \sum_{s} \sum_{t}\left[{ }^{\mathrm{A}} \mu_{i}^{r 0 \mathrm{~A}} \mu_{j}^{s r \mathrm{~A}} \mu_{k}^{t s} \mathrm{~A} \mu_{l}^{\alpha t}\left(E_{0 \mathrm{r}}+\hbar \omega\right)^{-1}\left(E_{0 s}+2 \hbar \omega\right)^{-1}\left(E_{0 t}+3 \hbar \omega\right)^{-1}\right. \\
& +{ }^{\mathrm{A}} \mu_{i}^{r 0 \mathrm{~A}} \mu_{j}^{s \mathrm{~A} A} \mu_{k}^{\alpha t \mathrm{~A}} \mu_{i}^{t s}\left(E_{0 r}+\hbar \omega\right)^{-1}\left(E_{0 s}+2 \hbar \omega\right)^{-1} \\
& \times\left(E_{\alpha t}-\hbar \omega\right)^{-1}+{ }^{\mathrm{A}} \mu_{i}^{\mathrm{r} O \mathrm{~A}} \mu_{j}^{t s \mathrm{~A}} \mu_{k}^{\alpha t \mathrm{~A}} \mu_{l}^{s r}\left(E_{0 \mathrm{r}}+\hbar \omega\right)^{-1} \\
& \times\left(E_{\alpha s}-2 \hbar \omega\right)^{-1}\left(E_{\alpha t}-\hbar \omega\right)^{-1}+{ }^{\mathrm{A}} \mu_{i}^{s \mathrm{~A}} \mu_{j}^{s s \mathrm{~A}} \mu_{k}^{\alpha t \mathrm{~A}} \mu_{i}^{r 0} \\
& \left.\times\left(E_{\alpha r}^{\prime}-3 \hbar \omega\right)^{-1}\left(E_{\alpha s}-2 \hbar \omega\right)^{-1}\left(E_{\alpha t}-\hbar \omega\right)^{-1}\right] .
\end{aligned}
$$

Here $\chi_{(i j) k}^{\alpha 0}$ can be identified with $\frac{1}{2}\left(\chi_{i j k}^{\alpha 0}+\chi_{j i k}^{\alpha 0}\right)$ and $T_{(j, k) l}^{\alpha 0}$ with $\frac{1}{6}\left(T_{i j k l}^{\alpha 0}+T_{i k j l}^{\alpha 0}+T_{j k i l}^{\alpha 0}+T_{j k k l}^{\alpha 0}+\right.$ $\left.T_{k i j i}^{\alpha 0}+T_{k j i l}^{\alpha 0}\right)$.

The rate equations as given above are valid for a rigid orientation of the laser beam with respect to both molecules. If the sample in question is fluid, a rotational average of the photon polarization vector $e$ has to be performed. The result will then hold for the 'rotating pair' case of two molecules A and B whose mutual orientation is fixed; this includes also the case of two chromphores $A$ and $B$ in one large rigid molecule. If the molecules $A$ and $B$ are free to change their mutual orientation, for example in a liquid or gas, a further average of all molecular tensors pertaining to $A$ is necessary and finally an average of the molecular tensors belonging to $B$. Here the sequence of the triple averaging is not important.

\section{Near-zone contributions in fluids}

The near-zone is defined by $R \ll \lambda=2 \pi c / \omega=2 \pi / k$, i,e, the distance between the molecules should be much smaller than the photon wavelength, a condition which results in a markedly enhanced bimolecular absorption rate. From a practical point of view, the near-zone corresponds to two closely spaced molecules without substantial wavefunction overlap. From a theoretical viewpoint, the matrix element phase factors reduce to

$$
\exp (-2 \mathrm{i} k \cdot \boldsymbol{R}) \approx 1 \approx \exp (-3 \mathrm{i} k \cdot \boldsymbol{R})
$$


which leads to considerably simplified calculations of the averaged rates. In this region the virtual photon coupling tensor also reduces to Coulombic coupling; hence it is not retarded, not dependent on $\omega, \omega_{\alpha 0}$ or $\omega_{\beta 0}$, and it is real:

$$
V_{(i j)}=\bar{V}_{(j)}=\left(4 \pi \varepsilon_{0} R^{3}\right)^{-1}\left(\delta_{i j}-3 \hat{R}_{i} \hat{R}_{j}\right)
$$

from which we can infer an $R^{-6}$ range dependence of the process rate.

The first rotational average of equations (8) and (9) is now obtained using standard procedures. These will hold per se for the case of a 'rotating pair'. The result for the (4.1) mechanism is:

$$
\begin{aligned}
\left\langle\Gamma^{(4,1)}\right\rangle=L / 35 & \left.\delta_{i k}-3 \hat{R}_{i} \hat{R}_{k}\right)\left(\delta_{j l}-3 \hat{R}_{j} \hat{R}_{l}\right) \\
& \times\left\{\left[T_{(m n n) i}^{\alpha 0} \bar{T}_{(m o o) j}^{\alpha 0}(6 \eta-3)+T_{(m n o) i}^{\alpha 0} \bar{T}_{(m n o) j}^{\alpha 0}(-3 \eta+5)\right]\right. \\
& \times \mu_{k}^{\beta 0} \bar{\mu}_{l}^{\beta 0}+\left[T_{(m n n) i}^{\beta 0} \bar{T}_{(m o o) j}^{\beta 0}(6 \eta-3)+T_{(m n o) i}^{\beta 0}\right. \\
& \left.\times \bar{T}_{(m n o) j}^{\beta 0}(-3 \eta+5)\right] \mu_{k}^{\alpha 0} \bar{\mu}_{l}^{\alpha 0}+2\left[T_{(m n n) i}^{\alpha 0} \bar{T}_{(m o o) j}^{\beta 0}(6 \eta-3)\right. \\
& \left.\left.+T_{(m n o) i}^{\alpha 0} \bar{T}_{(m n o) j}^{\beta 0}(-3 \eta+5)\right] \mu_{k}^{\beta 0} \bar{\mu}_{l}^{\alpha 0}\right\}
\end{aligned}
$$

where

$$
L=\rho_{f} I^{3}\left(64 \pi \hbar c^{3} \varepsilon_{0}^{5} R^{6}\right)^{-1}
$$

and the polarization parameter

$$
\eta=(e \cdot e)(\bar{e} \cdot \bar{e})=\frac{1}{2}[1+\cos (2 \alpha)]
$$

where $\alpha=0^{\circ}$ for linear and $\pm 90^{\circ}$ for circular polarization, and hence $\eta=1$ and 0 , respectively. Inspection of equation (16) reveals that it is not possible to obtain a zero value for the rate merely by choice of polarization; however, the terms connected with $(6 \eta-3)$ will be zero if an elliptical polarization of $\pm 45^{\circ}$ is employed.

The result for the $(3,2)$ mechanism is:

$$
\begin{aligned}
& \left\langle\Gamma^{(3,2)}\right\rangle=(L / 105)\left(\delta_{i k}-3 \hat{R}_{t} \hat{R}_{k}\right)\left(\delta_{j l}-3 \hat{R}_{j} \hat{R}_{l}\right) \\
& \times\left\{[ 4 \eta - 2 ] \left[\chi_{(\mathrm{mm}) i}^{\alpha 0} \bar{\chi}_{(\mathrm{no}) j}^{\alpha 0} S_{n k}^{\beta 0} \bar{S}_{o l}^{\beta 0}\right.\right. \\
& \left.+\chi_{(m m) i}^{\beta 0} \bar{X}_{(n o) j}^{\beta 0} S_{n k}^{\alpha 0} \bar{S}_{o l}^{\alpha 0}+2 \chi_{(m m) i}^{\alpha 0} \bar{X}_{(n o) j}^{\beta 0} S_{n k}^{\beta 0} \bar{S}_{o l}^{\alpha 0}\right] \\
& +[2 \eta-1]\left[\chi_{(m m) i}^{\alpha 0} \bar{X}_{(m)) j}^{\alpha 0} S_{o k}^{\beta 0} \bar{S}_{o l}^{\beta 0}+\chi_{(m m) t}^{\beta 0} \bar{X}_{(n n) j}^{\beta 0} S_{o k}^{\alpha 0} \bar{S}_{o l}^{\alpha 0}\right. \\
& \left.+2 \chi_{(m m) i}^{\alpha 0} \bar{\chi}_{(n n) j)}^{\beta 0} S_{o k}^{\beta 0} \bar{S}_{o l}^{\alpha 0}\right]+[8 \eta-4]\left[\chi_{(m n) !}^{\alpha 0} \bar{\chi}_{(n o) j}^{\alpha 0} S_{m k}^{\beta 0} S_{o l}^{\beta 0}\right. \\
& \left.+\chi_{(m n),}^{\beta 0} \bar{X}_{(n o) j}^{\beta 0} S_{m k}^{\alpha 0} \bar{S}_{o l}^{\alpha 0}+2 \chi_{(m n) !}^{\alpha 0} \bar{X}_{(n o) s}^{\beta 0} S_{m k}^{\beta 0} \bar{S}_{o l}^{\alpha 0}\right] \\
& +[4 \eta-2]\left[\chi_{(m n) i}^{\alpha 0} \bar{X}_{(0 o))}^{\alpha 0} S_{m k}^{\beta 0} \bar{S}_{n l}^{\beta 0}+\chi_{(m n) i}^{\beta 0} \bar{X}_{(o o) j}^{\beta 0} S_{m k}^{\alpha 0} \bar{S}_{n l}^{\alpha 0}\right. \\
& \left.+2 \chi_{(m n) i}^{\alpha 0} \bar{X}_{(o o) j}^{\beta 0} S_{m k}^{\beta 0} \bar{S}_{n l}^{\alpha 0}\right]+[-3 \eta+5]\left[\chi_{(m n) !}^{\alpha 0} \bar{X}_{(m)) j}^{\alpha 0} S_{o k}^{\beta 0} \bar{S}_{o l}^{\beta 0}\right. \\
& \left.+\chi_{(m n) i}^{\beta 0} \bar{X}_{(m n))}^{\beta 0} S_{o k}^{\alpha 0} \bar{S}_{o l}^{\alpha 0}+2 \chi_{(m n) i}^{\alpha 0} \bar{X}_{(m n) j}^{\beta 0} S_{o k}^{\beta 0} \bar{S}_{o l}^{\alpha 0}\right] \\
& +[-6 \eta+10]\left[\chi_{(m n) i}^{\alpha 0} \bar{X}_{(n o) j}^{\alpha 0} S_{o k}^{\beta 0} \bar{S}_{m i}^{\beta 0}+\chi_{(m n) i}^{\beta 0} \bar{X}_{(n o) j}^{\beta 0} S_{o k}^{\alpha 0} \bar{S}_{m l}^{\alpha 0}\right. \\
& \left.\left.+2 \chi_{(m n) i}^{\alpha 0} \bar{X}_{(n o) j}^{\beta 0} S_{o k}^{\beta 0} \bar{S}_{m i}^{\alpha 0}\right]\right\}
\end{aligned}
$$

which is non-zero for all polarization arrangements. With $\pm 45^{\circ}$ elliptical polarization, one may again effectively 'switch off' some of the rate contributions. 
Further averaging is needed to obtain the rate equations for free molecules. Specifically, molecular parameters relating to A and B must be averaged both over their mutual orientations and over their orientations with respect to the intermolecular displacement vector $\boldsymbol{R}$. Full details of this procedure, as applied to the two-photon bimolecular case, are given elsewhere (Andrews and Hopkins 1990). The results for the three-photon case are as follows:

$$
\begin{aligned}
& \left\langle\left\langle\left(\Gamma^{(4,1)}\right\rangle\right\rangle=(L / 525)\left\{\left[T_{(m n n) t}^{\alpha 0} \bar{T}_{(\text {moo) }}^{\alpha 0}(60 \eta-30)\right.\right.\right. \\
& \left.+T_{(m n o),}^{\alpha 0} \bar{T}_{(m n o) i}^{\alpha 0}(-30 \eta+50)\right]\left|\mu^{\beta 0}\right|^{2}+\left[T_{(m n n) i}^{\beta 0} \bar{T}_{(m o o) i}^{\beta 0}(60 \eta-30)\right. \\
& \left.+T_{(m n o) i}^{\beta 0} \bar{T}_{(m n o) i}^{\beta 0}(-30 \eta+50)\right]\left|\mu^{\alpha 0}\right|^{2} \\
& \left.+T_{(m n n) i}^{\alpha 0} \mu_{j}^{\alpha 0} \varepsilon_{m i j} \bar{T}_{(p o o) q}^{\beta 0} \mu_{r}^{\beta 0} \varepsilon_{p q r}(24 \eta-5)\right\} \\
& \left\langle\left\langle\left\langle\Gamma^{(3,2)}\right\rangle\right\rangle=(L / 7875)\left\{S _ { i j } ^ { \alpha 0 } \overline { S } _ { i j } ^ { \alpha 0 } \left[\chi_{(m m) k}^{\beta 0} \bar{\chi}_{(n n) k}^{\beta 0}(556 \eta-278)\right.\right.\right. \\
& +\chi_{(m m) k}^{\beta 0} \bar{\chi}_{(k n) n}^{\beta 0}(-168 \eta+84)+\chi_{(m n) k}^{\beta 0} \bar{\chi}_{(m n) k}^{\beta 0}(64 \eta+542) \\
& \left.+\chi_{(k m) m}^{\beta 0} \bar{\chi}_{(k n) n}^{\beta 0}(-21 \eta-63)+\chi_{(m n) k}^{\beta 0} \bar{X}_{(m k) n}^{\beta 0}(42 \eta+168)\right] \\
& +S_{t y}^{\alpha 0} \bar{S}_{j z}^{\alpha 0}\left[\chi_{(m m) k}^{\beta 0} \bar{\chi}_{(n n) k}^{\beta 0}(-4 \eta+2)\right. \\
& +\chi_{(m m) k}^{\beta 0} \bar{\chi}_{(k n) n}^{\beta 0}(12 \eta-6)+\chi_{(m n) k}^{\beta 0} \bar{\chi}_{(m n) k}^{\beta 0}(-\eta-3) \\
& \left.+\chi_{(k m) m}^{\beta 0} \bar{\chi}_{(k n) n}^{\beta 0}(14 \eta+42)+\chi_{(m n) k}^{\beta 0} \bar{\chi}_{(m k) n}^{\beta 0}(-11 \eta-33)\right] \\
& +S_{t i}^{\alpha 0} \bar{S}_{j j}^{\alpha 0}\left[\chi_{(m m) k}^{\beta 0} \bar{\chi}_{(n n) k}^{\beta 0}(-4 \eta+2)\right. \\
& +\chi_{(m m) k}^{\beta 0} \bar{\chi}_{(k n) n}^{\beta 0}(12 \eta-6)+\chi_{(m n) k}^{\beta 0} \bar{\chi}_{(m n) k}^{\beta 0}(-\eta-3) \\
& +\chi_{(k m) m}^{\beta 0} \bar{\chi}_{(k n) n}^{\beta 0}(-11 \eta-33) \\
& \left.+\chi_{(m n) k}^{\beta 0} \bar{\chi}_{(m k) n}^{\beta 0}(14 \eta+42)\right]+[\alpha \leftrightarrow \beta] \\
& +\varepsilon_{i j k} \varepsilon_{o p q}\left[\chi _ { ( m m ) i } ^ { \alpha 0 } \overline { S } _ { j k } ^ { \alpha 0 } \left[\bar{\chi}_{(n n) o}^{\beta 0} S_{p q}^{\beta 0}(50 \eta-25)\right.\right. \\
& \left.+\bar{\chi}_{(n o) p}^{\beta 0} S_{n q}^{\beta 0}(-100 \eta+50)\right] \\
& +\chi_{(m i)}^{\alpha 0} \bar{S}_{m k}^{\alpha 0}\left[\bar{\chi}_{(n n) 0}^{\beta 0} S_{p q}^{\beta 0}(-220 \eta+110)\right. \\
& +\bar{\chi}_{(\text {on }) n}^{\beta 0} S_{p q}^{\beta 0}(95 \eta-29)+\bar{\chi}_{(n o) p}^{\beta 0} S_{n q}^{\beta 0}(-195 \eta+325) \\
& \left.+\bar{\chi}_{(n o) p}^{\beta 0} S_{q n}^{\beta 0}(95 \eta-29)\right] \\
& +\chi_{(m i) j}^{\alpha 0} \bar{S}_{k m}^{\alpha 0}\left[\bar{\chi}_{(n n) o}^{\beta 0} S_{p q}^{\beta 0}(40 \eta-20)\right. \\
& +\bar{\chi}_{(o n) n}^{\beta 0} S_{p q}^{\beta 0}(-83 \eta+73)+\bar{\chi}_{(\text {no }) p}^{\beta 0} S_{n g}^{\beta 0}(15 \eta-25) \\
& \left.+\bar{\chi}_{(n 0) p}^{\beta 0} S_{q \pi}^{\beta 0}(-97 \eta+87)\right] \\
& +\chi_{(i m) m}^{\alpha 0} \bar{S}_{j k}^{\alpha 0}\left[\bar{\chi}_{(n n) o}^{\beta 0} S_{p q}^{\beta 0}(40 \eta-20)\right. \\
& +\bar{\chi}_{(\text {on }) n}^{\beta 0} S_{p q}^{\beta 0}(-97 \eta+87)+\bar{\chi}_{(n o) p}^{\beta 0} S_{n g}^{\beta 0}(15 \eta-25) \\
& \left.\left.\left.+\bar{\chi}_{(n o) p}^{\beta 0} S_{q n}^{\beta 0}(-83 \eta+73)\right]\right]\right\}
\end{aligned}
$$

where $[\alpha \leftrightarrow \beta]$ denotes a term derived from the preceding one by interchange of the superscript labels, thereby signifying a contribution in which the roles of A and B are themselves exchanged. 


\section{Longer range contributions in fluids}

At longer distances where the condition $R \ll \lambda$ does not hold, the simplifications given in section 3 do not persist and the rotational average(s) lead to very complicated results. Fortunately the most important features, especially the dependence on molecular separation $R$ and polarization, can be recovered without reference to the detail of the average. In the following paragraphs we will distinguish between the first two (diagonal) contributions to equations (8) and (9) which may be signified by $A A \mid B B$ and $\mathrm{BB} \mid \mathrm{AA}$ and the interference contributions $\mathrm{AB} \mid \mathrm{BA}$.

\subsection{Diagonal terms}

The first two terms in equations (8) and (9) are not associated with a phase factor and hence average to expressions very similar to their analogues, equations (16) and (19) for the rotating pair and (20) and (21) for free molecules. However, the fully retarded form of coupling must now be employed and this is reflected in the appearance of a term $V_{(i j)} \bar{V}_{(i j)}$, representing an excitation transfer function, after the triple average. For the $(4,1)$ mechanism it leads to

$$
\begin{aligned}
& V_{(i j)}\left(\omega_{\alpha 0}, R\right) \bar{V}_{(i j)}\left(\omega_{\alpha 0}, R\right)=2 Q(0, R)\left[3+K_{\mathrm{A}}^{2} R^{2}+K_{\mathrm{A}}^{4} R^{4}\right] \\
& V_{(i j)}\left(\omega_{\beta 0}, R\right) \bar{V}_{(i j)}\left(\omega_{\beta 0}, R\right)=2 Q(0, R)\left[3+K_{\mathrm{B}}^{2} R^{2}+K_{\mathrm{B}}^{4} R^{4}\right]
\end{aligned}
$$

with $K_{\mathrm{A}}=\omega_{\alpha} / c, K_{\mathrm{B}}=\omega_{\beta 0} / c$ and

$$
Q(K, R)=\left(4 \pi \varepsilon_{0} R^{3}\right)^{-1} \exp (\mathrm{i} K R) \text {. }
$$

Equations (22) and (23) can be identified with the familiar transfer functions $A\left(K_{A}, R\right)$ and $A\left(K_{\mathrm{B}}, R\right)$. These give rise to identifiable retardation features in the theory of intermolecular electronic excitation transfer and as such have been extensively discussed elsewhere (Andrews and Juzeliunas 1992). For the $(3,2)$ mechanism $\left(K_{\mathrm{A}}-k\right)$ and $\left(K_{\mathrm{B}}-k\right)$ are used in place of $K_{\mathrm{A}}$ and $K_{\mathrm{B}}$.

\subsection{Interference terms}

The last terms in equations (8) and (9) are quantum mechanical interference terms which arise only under certain selection rule conditions, as we discuss in section 5 . Since these terms include phase factors, they have to be subjected to so-called phased averaging (Andrews and Harlow 1984).

The first (phased) average leads to a rotating pair rate which is expressible as a sum of terms involving the spherical Bessel functions $j_{m}$ with $0 \leqslant m \leqslant 6$. Specifically, these are $j_{m}(3 k R)$ for the $(4,1)$ mechanism and $j_{m}(2 k R)$ for the $(3,2)$ mechanism. The terms which multiply each $j_{m}$ comprise products of scalar parameters associated with molecular response and polarization conditions. These scalars are derived through contractions both of the molecular and the polarization tensors with isotropic tensors of rank $(6+m)$. In turn, the isotropic tensors are expressible as products of Kronecker delta tensors (together with one Levi-Civita antisymmetric tensor in the odd $m$ cases). It is worth noting that for $k R \ll 1$ the term with $j_{0} \approx 1$ strongly dominates the rate leading to the near-zone behaviour discussed in section 3 . For medium-range distances $0 \ll k R<1$ it is only the $j_{1}$ term which can give any significant additional contribution. 
For any plane or circular polarization, the polarization parameters acquire only two different values, 1 and $\eta$, for even $m$, but $0, \zeta=\hat{k} \cdot(e \wedge \bar{e})$ and $\eta \zeta$ for odd $m$ (note that $\left.\eta=1+\zeta^{2}\right)$. It is significant that in the latter, odd $m$, case all polarization parameters are zero for linear polarization and are non-zero but with opposite signs for left- and right-handed circular polarization. The conclusion is that circular dichroism appears when a rotating pair of molecules or chromophores is irradiated under the condition $k R \gg 0$. It is however a very weak effect which would most likely require use of polarization modulation techniques sor its detection. In passing we note that exactly the same considerations apply to one- and two-photon bimolecular absorption.

For free molecules the corresponding results are obtained from further rotational averages discussed in the previous section. Here the picture changes: for odd $m$ these (non-phased) averages result in a contraction of each coupling tensor product $V_{(i j)} \bar{V}_{(k l)}$ with a combination of a Levi-Civita tensor and one or three unit vectors $\hat{\boldsymbol{R}}$, which is easily shown to yield a zero result. So contributions from even $m$ alone arise, and no circular dichroism is possible. For even $m$ the coupling tensor combination $V_{(i j)} \bar{V}_{(k l)}$ is contracted as follows: for $m=0$ with three Kronecker deltas ' $\delta \delta \delta$ ', for $m=2$ additionally with ' $\delta \delta \hat{R} \hat{R}$ ', for $m=4$ also with ' $\delta \hat{R} \hat{R} \hat{R} \hat{R}$ ', and for $m=6$ furthermore with ' $\hat{R} \hat{R} \hat{R} \hat{R} \hat{R} \hat{R}$ '. It is evident that apart from equations (22) and (23) only the following four excitation transfer functions arise $f$ :

$$
\begin{aligned}
& V_{(i j)}\left(\omega_{\beta 0}, R\right) \bar{V}_{(i j)}\left(\omega_{\alpha 0}, R\right) \\
&= 2 Q\left(K_{\mathrm{B}}-K_{\mathrm{A}}, R\right)\left[3-3 \mathrm{i}\left(K_{\mathrm{B}}-K_{\mathrm{A}}\right) R+\left(3 K_{\mathrm{A}} K_{\mathrm{B}}-K_{\mathrm{A}}^{2}-K_{\mathrm{B}}^{2}\right) R^{2}\right. \\
&\left.-\mathrm{i}\left(K_{\mathrm{B}}-K_{\mathrm{A}}\right) K_{\mathrm{A}} K_{\mathrm{B}} R^{3}+K_{\mathrm{A}}^{2} K_{\mathrm{B}}^{2} R^{4}\right] \\
& V_{(i i)}\left(\omega_{\beta 0}, R\right) \bar{V}_{(j j)}\left(\omega_{\alpha 0}, R\right)=2 Q\left(K_{\mathrm{B}}-K_{\mathrm{A}}, R\right)\left[K_{\mathrm{A}}^{2} K_{\mathrm{B}}^{2} R^{4}\right]
\end{aligned}
$$

associated with $j_{0}, j_{2}, j_{4}$ and $j_{6}$, and

$$
\begin{aligned}
& \begin{aligned}
V_{(i j)}\left(\omega_{\beta 0}, \boldsymbol{R}\right) & \bar{V}_{(i k)}\left(\omega_{\alpha 0}, \boldsymbol{R}\right) \hat{R}_{j} \hat{R}_{k} \\
= & V_{(i j)}\left(\omega_{\beta 0}, \boldsymbol{R}\right) \bar{V}_{(k l)}\left(\omega_{\alpha 0}, \boldsymbol{R}\right) \hat{R}_{i} \hat{R}_{j} \hat{R}_{k} \hat{R}_{l} \\
= & V_{(j)}\left(\omega_{\beta 0}, \boldsymbol{R}\right) \bar{V}_{(k l)}\left(\omega_{\alpha 0}, \boldsymbol{R}\right) \hat{R}_{i} \hat{R}_{j} \hat{R}_{k} \hat{R}_{l} \hat{R}_{m} \hat{R}_{m} \\
= & 4 Q\left(K_{\mathrm{B}}-K_{\mathrm{A}}, R\right)\left[1-\mathrm{i}\left(K_{\mathrm{B}}-K_{\mathrm{A}}\right)+K_{\mathrm{A}} K_{\mathrm{B}} R^{2}\right]
\end{aligned} \\
& V_{(i j)}\left(\omega_{\beta 0}, \boldsymbol{R}\right) \bar{V}_{(k k)}\left(\omega_{\alpha 0}, \boldsymbol{R}\right) \hat{R}_{1} \hat{R}_{j}=4 Q\left(K_{\mathrm{B}}-K_{\mathrm{A}}, R\right)\left[K_{\mathrm{A}}^{2} R^{2}-\mathrm{i} K_{\mathrm{A}}^{2} K_{\mathrm{B}} R^{3}\right]
\end{aligned}
$$

associated with $j_{2}, j_{4}$ and $j_{6}$. Once again for the $(3,2)$ mechanism $\left(K_{\mathrm{A}}-k\right)$ and $\left(K_{\mathrm{B}}-k\right)$ have to be used in place of $K_{\mathrm{A}}$ and $K_{\mathrm{B}}$. All of the above transfer functions also arise in connection with two-photon absorption and Raman bimolecular processes (Andrews and Blake 1990).

\subsection{Range dependence}

In order to illustrate the range dependence, we concentrate on the case of two identical molecules with identical excited states, i.e. $\mathrm{A}=\mathrm{B}$ and $E_{\alpha 0}=E_{\beta 0}$, setting $K_{\mathrm{A}}=K_{\mathrm{B}}=K$ and $3 k=2 K$. The argument of the spherical Bessel functions is $3 k R=2 K R$ in the $(4,1)$ case and $2 k R=\frac{4}{3} K R$ in the $(3,2)$ case. Also in equations (25)-(28), the $Q$ function defined by equation (24) now has an argument $Q(K-K, R)=Q(0, R)$ and hence becomes real. Although there remains an imaginary term in equation (28), it does not 
contribute to the eventual rate equations as only the real part of the interference terms has to be taken (see equations (8) and (9)). Hence, as is necessary, the final result is an entirely real quantity.

Under these conditions, the excitation transfer functions given by equations (22), (23) and (25) now become equal, so that only four different transfer functions arise. Invoking the $R^{-6}$ dependence of $Q(0, R)$, they may be displayed as:

$$
\begin{aligned}
& A(K, R)=2\left(4 \pi \varepsilon_{0}\right)^{-2}\left(3+K^{2} R^{2}+K^{4} R^{4}\right) / R^{6} \\
& A^{\prime}(K, R)=4\left(4 \pi \varepsilon_{0}\right)^{-2} K^{4} / R^{2} \\
& A^{\prime \prime}(K, R)=4\left(4 \pi \varepsilon_{0}\right)^{-2}\left(1+K^{2} R^{2}\right) / R^{6} \\
& A^{\prime \prime \prime}(K, R)=4\left(4 \pi \varepsilon_{0}\right)^{-2} K^{2} / R^{4}
\end{aligned}
$$

As given above, these equations are valid for the $(4,1)$ case; for the $(3,2)$ mechanism, $K$ is simply replaced by $K-k=\frac{1}{3} K$. The transfer function $A(K, R)$ has already been defined in section 4.1; it is also included in the diagonal terms. The interference terms comprise $A(K, R) j_{0}, A^{\prime}(K, R) j_{0}$ and for $m=2,4,6$ also $A^{\prime \prime}(K, R) j_{m}$ and $A^{\prime \prime \prime}(K, R) j_{m}$. It is therefore evident that the rate contributions associated with $A^{\prime \prime}$ and $A^{\prime \prime \prime}$ contribute very little if $k R<1$; due to the higher orders of $j_{m}$ with which they are associated, they are important only for large distances.

Generally the rate contribution governed by the transfer function $A$ will exceed all other terms, although for certain distances in the intermediate range the range dependence may be marginally modified by the additional contributions from the $A \times j_{0}$ and $A^{\prime} \times j_{0}$ cross-terms, if allowed by the selection rules. Hence we can conclude that it is entirely justified to neglect any contributions to the overall rate of absorption from transfer functions other than $A$. This argument applies to the three-photon process under consideration here, and for similar reasons to one-, two- or any other multiphoton bimolecular processes.

\section{Selection rules}

For determining the selection rules one has to take account of the symmetry properties of the molecular tensors which feature in the rate equations. For the rotationally averaged rate equations, the symmetry of the Levi-Civita tensors must also be taken into account. Equations $(8)$ and $(9)$ show that in the $(4,1)$ process the $T$ tensor and the transition dipole moment $\mu$ arise, in the $(3,2)$ process the $\chi$ and $S$ tensors. Both equations show a common principal structure which may be abbreviated to $T T \mid \mu \mu$ for the $(4,1)$ case and $\chi \chi \mid S S$ for the $(3,2)$ case. Both also comprise three terms which differ with respect to the tensor ordering; as above they may be signified by $\mathrm{AA}|\mathrm{BB}, \mathrm{BB}| \mathrm{AA}$ (diagonal terms) and $\mathrm{AB} \mid \mathrm{BA}$ (interference terms).

Using irreducible tensor methods, one can assign weights to the tensors: $\mu$ transforms as a polar vector and so possesses weight $(-1) ; S$ transforms as a second rank tensor and has weights $(+2),(+1),(+0) ; \chi$ is a third rank tensor symmetric in two indices, and has weights $(-3),(-2),(-1)$; the fourth rank tensor $T$ has weights $(+4)$, $(+3),(+2),(+1),(+0)$. The transitions in $A$ and $B$ may now be assigned to an irreducible representation of each applicable point group. Different combinations of the tensor weights can transform under the various irreducible representions of each point group, and the various possibilities are listed elsewhere (Andrews 1990). A transition in molecule $A$ whose assigned irreducible representation contains for example weight 
$(+0)$ or $(+1)$ or $(+2)$ is termed $A^{+} \overline{(012)}$. It now becomes evident that in both mechanisms for bimolecular three-photon absorption, assuming that $A$ and $B$ are either chemically different or are at least promoted to different excited states, then for the majority of possible cases a single diagonal term $\mathrm{AA} \mid \mathrm{BB}$ (or $\mathrm{BB} \mid \mathrm{AA}$ ) alone is present. The selection rules thus deduced are

$$
\begin{aligned}
& (4,1) \text { mechanism: } A^{+} \overline{(01234)}, B^{-}(1) \\
& (3,2) \text { mechanism: } A^{-} \overline{(123)}, B^{+} \overline{(012)} \text {. }
\end{aligned}
$$

In the case of the $(4,1)$ mechanism the above rule $(33)$ relates to the first term of equation (8), and a counterpart in which the roles of $\mathrm{A}$ and $\mathrm{B}$ are exchanged applies to the second term. The third $\mathrm{AB} \mid \mathrm{BA}$ term can clearly arise only when the transitions of both A and B satisfy both conditions (33). It may also be noted that the further symmetry constraints on this term which arise in the case of free molecules necessitate satisfaction of the condition ${ }^{+}(1) \wedge^{-}(1)$ by both molecules, where the symbol $\wedge$ denotes the logical AND operator. This amounts to a requirement for both $\alpha$ and $\beta$ excited state representations to span both translation and rotation components. For the $(3,2)$ mechanism whose rate results from equation (9), similar arguments apply with respect to the conditions (34). Here, in the case of free molecules, the interference term can only persist if both molecular transitions fulfil the condition $+\overline{(012)} \wedge-\overline{(12)}$.

For both mechanisms it is transparent that appearance of the interference term necessitates the presence of contributions from all three terms $\mathrm{AA}|\mathrm{BB}, \mathrm{BB}| \mathrm{AA}$ and AB $\mid$ BA. In a previous paper (Andrews and Bittner 1992) the selection rules (33) and (34) have been applied to molecular point groups and a comprehensive table of allowed transitions given.

\section{Discussion}

One of the first considerations has to be the likelihood of observing bimolecular effects in connection with the intrinsically weak process of three-photon absorption. A number of ameliorating factors needs to be considered. First, it has been demonstrated in several earlier treatments of bimolecular absorption that the rate displayed by pairs of molecules in close proximity should approach the magnitude of a single-centre rate: such arguments apply with equal force to the three-photon case. It should be borne in mind that the assumption in the detailed theory of electric dipole coupling is appropriate only when the molecular separation exceeds the molecular dimensions; this however is consistent with the neglect of wavefunction overlap. A second and more positive factor in favour of three-photon bimolecular excitation is the possibility of selecting a chemical system in which the selection rules for one or both of the mechanisms militate against any other kind of excitation at the laser wavelength employed.

Thirdly the cubic dependence of the system rate on irradiance has a special significance with the continued trend towards lasers with higher levels of output. The fifth-order perturbation theory which underlies the three-photon bimolecular mechanisms discussed above is valid for beam intensities $I$ up to those levels $I \sim I_{\mathrm{M}}=$ $10^{18 \pm 4} \mathrm{~W} \mathrm{~m}^{-2}$ where the associated electric fields approach the magnitude of the internal molecular fields associated with bonding (Eberly et al 1987). Below these intensity levels the three-photon rate will be smaller than the corresponding one-photon rate by a factor typically of the order $\left(I / I_{\mathrm{M}}\right)^{3}$, if both processes are permitted by the 
selection rules. Current laser instrumentation allows the production of focused intensity levels for which $\left(I / I_{\mathrm{M}}\right)^{3}$ is only one or two orders of magnitude below unity, such that detection of the three-photon bimolecular process is perfectly viable and the perturbative treatment of the theory remains fully appropriate.

Fourthly, there is the possibility of exploiting near-resonance conditions in order to obtain rate enhancement, typically by several orders of magnitude. Indeed, more such possibilities arise in the three-photon case than in processes involving fewer photons, as illustrated in figure 1 (note that the broken and dotted lines correspond to fully resonant levels which should be avoided if their energy equals multiples of the photon energy $\hbar \omega$ ). One additional point to consider is the possibility of two-colour (or three-colour) excitation, which although not treated in the theory described in this paper clearly carries many further possibilities for rate enhancement by the exploitation of resonance amplification.

Possible chemical systems in which bimolecular absorption might be characterized have been discussed at length in our recent paper (Andrews and Bittner 1992). Although a few somewhat esoteric cases were singled out to exemplify the three-photon case, in fact any of the schemes we discussed for the one-photon case would also be viable for three-photon study, given of course the appropriate longer-wavelength laser. More significantly, any of the numerous experimental instances of cooperative one-photon absorption would also be appropriate for three-photon study. Of the various means by which such a process might be observed, obvious candidates are laser-induced fluorescence or ionization detection, or a scheme involving optical-optical double resonance. A more novel possibility, particularly appropriate for systems with relatively discrete energy levels, would be to look for generation of a $\left(\frac{3}{2}\right)$ harmonic of the input frequency, produced by superradiant decay from the states populated by the excitation scheme.

\section{Acknowledgement}

One of us (AMB) gratefully acknowledges financial support from the Deutscher Akademischer Austauschdienst (DAAD).

\section{Appendix. Summing the matrix element contributions}

Summing the matrix element contributions associated with the numerous time-ordered diagrams of which figures $2(a)$ and $(b)$ are representative members entails collecting together all terms with a combination of transition moment components which is the same for both molecule $A$ and for $B$. Each term has a denominator expressed as a product of four factors; each factor is the difference of the intermediate (system plus radiation) state energy from the initial state energy. In cases where the virtual photon creation and annihilation events are not temporally adjacent, more than one of these factors will entail the virtual photon energy. The use of partial fractions and the overall energy conservation relation (4) nonetheless enables the virtual photon energy to be eliminated from every denominator save one which is of the form ( $\left.E_{\alpha 0} \pm \hbar c \kappa\right)$ or $\left(E_{\beta 0} \pm \hbar c \kappa\right)$. Use of equation (10) enables pairs of similar terms involving these factors to be added and recast in terms of the virtual photon (retarded resonance electric dipole-electric dipole) coupling tensor. Finally by introducing the molecular response 
tensors defined by $(11),(12)$ and $(13)$, the overall results for the $(4,1)$ and $(3,2)$ matrix elements are obtained:

$$
\begin{aligned}
& M^{(4,1)}=\mathrm{i}\left[\left(n \hbar \omega /\left(2 \varepsilon_{0} V\right)\right]^{3 / 2} e_{\imath} e_{j} e_{k}\right. \\
& \times\left\{T_{(j i k) l}^{\alpha 0} \mu_{m}^{\beta 0} V_{(l m)}\left(\omega_{\beta 0}, \boldsymbol{R}\right) \exp \left(3 \mathrm{i} \boldsymbol{k} \cdot \boldsymbol{R}_{\mathrm{A}}\right)\right. \\
& \left.+T_{(j i k) t}^{\beta 0} \mu_{m}^{\alpha 0} V_{(l m)}\left(\omega_{\alpha 0}, \boldsymbol{R}\right) \exp \left(3 \mathrm{i} k \cdot \boldsymbol{R}_{\mathrm{B}}\right)\right\} \\
& M^{(3,2)}=\mathrm{i}\left[\left(n \hbar \omega /\left(2 \varepsilon_{0} V\right)\right]^{3 / 2} e_{i} e_{j} e_{k}\right. \\
& \times\left\{\chi_{(i) l}^{\alpha 0} S_{k m}^{\beta 0} V_{(l m)}\left(\omega_{\beta 0}-\omega, \boldsymbol{R}\right) \exp \left[3 \mathrm{i} \boldsymbol{k} \cdot\left(\boldsymbol{R}_{\mathrm{A}}+\boldsymbol{R}\right)\right]\right. \\
& \left.+\chi_{(i j)\}}^{\beta 0} S_{k m}^{\alpha 0} V_{(I m)}\left(\omega_{\alpha 0}-\omega, \boldsymbol{R}\right) \exp \left[3 \mathrm{i} \boldsymbol{k} \cdot\left(\boldsymbol{R}_{\mathrm{B}}-\boldsymbol{R}\right)\right]\right\}
\end{aligned}
$$

\section{References}

Andrews D L 1990 Spectrochim. Acta $46 \mathrm{~A} 871$

Andrews D L and Bittner A M 1992 Chem. Phys. 1651

Andrews D L and Blake N P $1990 \mathrm{~J}$. Mod. Opt. 37701

Andrews D L, Craig D P and Thirunamachandran T 1989 Int. Rev. Phys. Chem. 8339

Andrews D L and Harlow M J 1984 Phys. Rev. A 292796

Andrews D L and Hopkins K P 1990 Adv. Chem. Phys. 7739

Andrews D L and Juzeliūnas G 1992 J. Chem. Phys. 966606

Craig D P and Thirunamachandran T 1984 Adv. Quantum Chem. 1697

Eberly J H, Maine P, Strickland D and Mourou G 1987 Laser Focus 23 (10) 84

Hudis E, Ben-Aryeh Y and Oppenheim U P 1991 Phys. Rev. A 433631

— 1992 J. Quant. Spectrosa. Radiat. Transfer 47319

Power E A and Zienau S 1959 Phil. Trans. R Soc, A 251427

Schipper P E 1981 Chem. Phys. 57105

Wallace R 1966 Mol. Phys. 11457

Woolley R G 1971 Proc. R. Soc. A 321557 